\title{
Multiple Impaction in Mandible: A Case Report of Kissing Molars
}

\author{
Dr. Kavya Priya $\mathrm{T}^{1}$, Dr. Vinayak Gourish Naik ${ }^{2 *}$, Dr. Shiva Kumar H. R ${ }^{3}$, Dr. Kirthi Kumar Rai, \\ $\mathrm{MDS}^{4}$ \\ ${ }^{\mathrm{T}}$ Lecturer, Department of Oral \& Maxillofacial Surgery, Bapuji Dental College and Hospital, \\ Davangere 577004, Karnataka, India \\ ${ }^{2}$ Reader, Department Of Oral \& Maxillofacial Surgery, Bapuji Dental College \& Hospital, Davangere- 577004, \\ Karnataka, India \\ ${ }^{3}$ Professor, Department of Oral \& Maxillofacial Surgery, Bapuji Dental College and Hospital, Davangere 577004, \\ Karnataka, India \\ ${ }^{4}$ Professor \& Head of Department, Department of Oral \& Maxillofacial Surgery, Bapuji Dental College and Hospital, \\ Davangere 577004, Karnataka, India
}

\section{*Corresponding Author}

Dr. Vinayak Gourish Naik

\section{Article History}

Received: 19.02 .2020

Accepted: 26.02 .2020

Published: 08.03.2020

\begin{abstract}
Kissing molars' is an interesting occurrence and also rare. Von Hoof described this phenomenon for the first time in 1973. He described it as two impacted molars with occlusal surface contacting each other with their roots in opposite direction in a single dental follicle. Literature has reported cases where the kissing molars involve second and third molar or third molar and supernumerary fourth molar. Kissing molars involving first and second molar is rare. Most often Cone Beam Computed Tomography (CBCT) is required to assess the close relationship of the impacted teeth with the inferior alveolar canal to avoid paraesthesia. Some authors have reported its occurrence in patients diagnosed with mucopolysaccharidosis and also as isolated cases. Greater importance should be given to treatment planning with thorough understanding of surrounding anatomy. Herewith we would like to report a case of unilateral kissing molars involving impacted first and second mandibular molar.
\end{abstract}

Keywords: Impacted molars, Mandibular Molar, Kissing molars, Mucopolysaccharidosis.

\section{INTRODUCTION}

Impaction of a second permanent molar is an unusual occurrence with an incidence of $0.03 \%$ to $0.21 \%$ in the mandible. Impaction of first permanent molar is even rarer, with incidence of less than $0.01 \%$ for the mandibular first molar [1]. R.F. Van Hoof described in 1973 the term "kissing molars" as two impacted molars with occlusal surface contacting each other in a single follicular space [2]. Literature consists of reports on kissing molars involving second molar with third molar or third molar with supernumerary fourth molar in the mandible, however there are no case reports of rosette formation that involves first and second mandibular molar. We present a rare case of kissing molars in the mandible involving first and second molar that exists in a synchronous impaction of all unilateral molars and also simultaneous impaction of second and third molars in the contralateral quadrant. Managing multiple impacted molars is a difficult task because of unknown aetiology and has no set standard treatment plan. Literature suggests therapies such as surgical removal, surgical up righting, transplantation and surgical-orthodontic approach which would be based on individual case and not a generalised management option [1, 3]. Analysing the ensuing complications such as paraesthesia is important because of the proximity to inferior alveolar nerve, other complications include dry socket, trismus, swelling and pain [4]. Investigations include panoramic radiographs and CBCT for establishing the exact position and distance between the molars and the inferior alveolar nerve. Kissing molars can be seen in isolation or associated with dentigerous cyst and mucopolysaccharoidosis [5].

Copyright @ 2020: This is an open-access article distributed under the terms of the Creative Commons Attribution license which permits unrestricted use, distribution, and reproduction in any medium for non commercial use (NonCommercial, or CC-BY-NC) provided the original author and source are credited. 


\section{CASE Report}

A 21 year old patient reported to the Department of Oral and Maxillofacial Surgery, Bapuji Dental College \& Hospital, with a chief complaint of missing teeth in the posterior lower jaw since 8 years and wanted dental rehabilitation for the same. His medical history revealed that the patient had delayed speech for which he received speech therapy at the age of 6 years but without much improvement. He also had a previous hospital admission for a laparoscopic surgery for cryptorchidism at the age of 21 years. Patient's father revealed that the patient doesn't communicate well with the family members and also doesn't socialise. His systematic status was normal. Local examination showed a mesencephalic, brachyfacial, convex profile with reduced midfacial height and deep mentolabial sulcus. He had depressed nasal bridge, hypertelorism, large nose and large lower lip. Though the general facial features suggest mucopolysaccharidosis, further investigation was needed to confirm the same however, the patient and his parents were not willing and not co-operative for further investigations regarding the metabolic disorder. The patient had achieved required milestones and had normal intellect as others for the specific age. Intraorally periodontal status was good. Mesiolingual rotated mandibular bilateral second premolars, Distopalatal rotated maxillary left lateral incisor, missing molars w.r.t mandibular right first, second and third molar, mandibular left second and third molar, maxillary right canine and third molar \& maxillary left third molar (Fig 1 and 2).

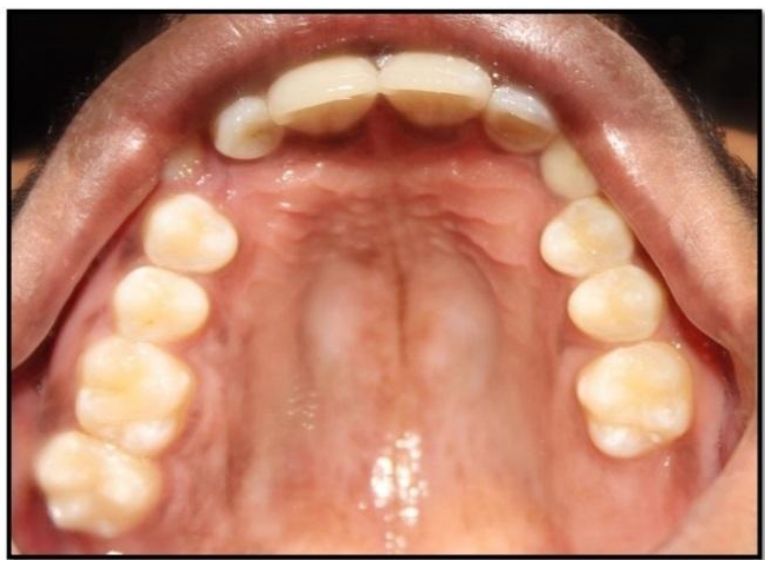

Fig-1: Maxillary arch showing missing bilateral third molars, right second molar and right canine

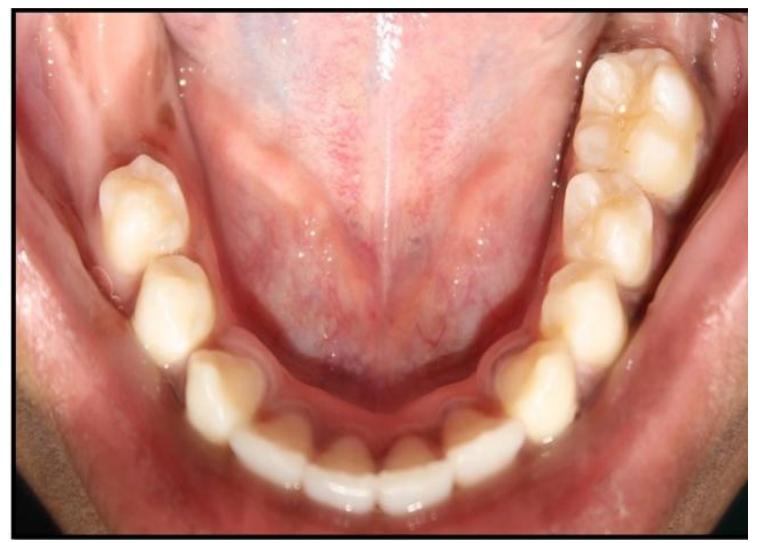

Fig-2: Mandibular arch showing all three missing molars on right side and missing second and third molars on left side

Panoramic radiograph (Fig-3) revealed horizontal impaction of mandibular left third molar and vertical impaction of second molar both within a single dental follicle but not in a typical rosette formation; impacted mandibular right first and second molars within a single dental follicle in a typical rosette formation i.e. occlusal surfaces facing each other with roots facing opposite to each other; mesioangular impaction of mandibular right third molar; vertical impaction of left maxillary third molar; vertical impaction of right maxillary canine; mesioangular impaction of right maxillary third molar. CBCT showed the exact position and distance of the roots of kissing molars from the inferior alveolar nerve (Fig-3a and Fig-3b). 


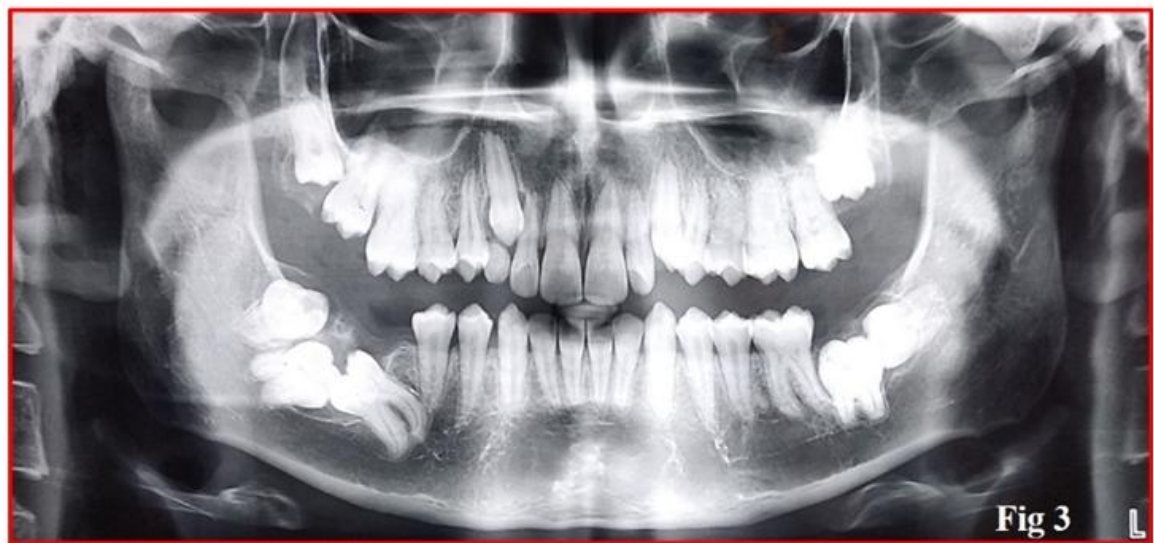

Fig-3: OPG showing right mandibular kissing molars, impacted third molar; impacted left mandibular second and third molar; bilateral impacted maxillary third molars and right impacted canine

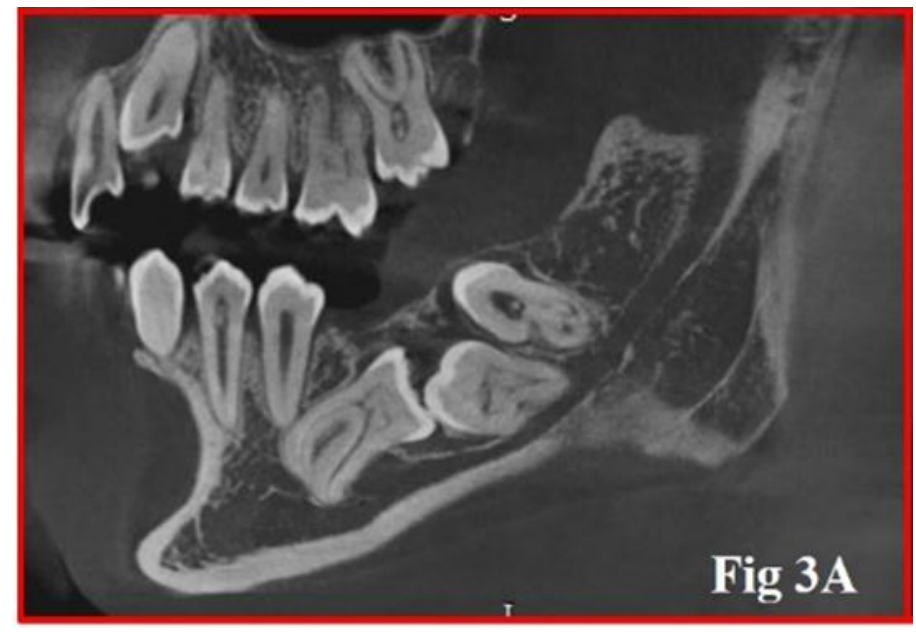

Fig-3a: CBCT axial section showing the 'rosette formation' between mandibular right first and second molar

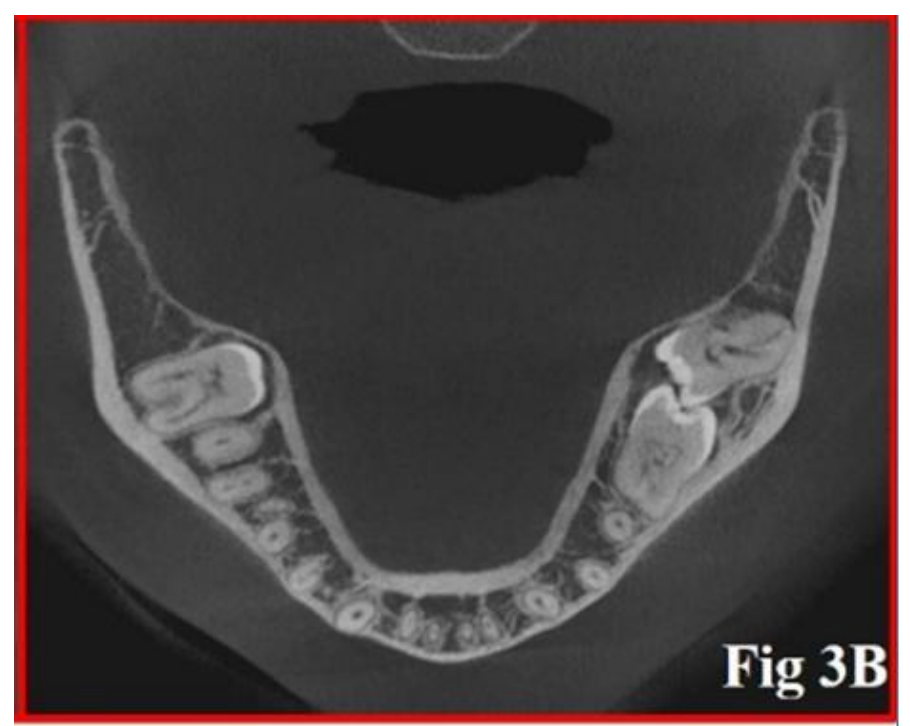

Fig-3b: CBCT sagittal section showing the proximity of mandibular right second and third molar to inferior alveolar nerve

Treatment plan included surgical removal of bilateral maxillary third molars, surgical exposure of right maxillary canine for orthodontic uprighting due to its favourable position for the same, surgical removal of left mandibular third molar making space for impacted second molar to erupt or for uprighting at a later date, surgical removal of right mandibular second and third molars leaving behind first molar giving it a chance and a duration of 6 months to erupt and consider surgical removal 6 months later in case it doesn't erupt since its position is not favourable for orthodontic up-righting. Surgical removal of right mandibular second molar was considered ideal since it was in an unfavourable position to erupt or for orthodontic up-righting and interlocked on the occlusal surface of first molar. 
Under general anaesthesia, a triangular flap was raised from the region of second premolar to third molar. Deroofing in the region of third molar exposed both the cusps of third molar, buccal bone guttering was done to expose the third molar till its Cementoenamel junction after which a Coupland elevator was used to deliver the tooth out. Buccal guttering was extended anteriorly until the crown and furcation of the second molar was exposed, the tooth was sectioned vertically and the two fragments were elevated separately following which the distal cusp of first molar was exposed (Fig-4).

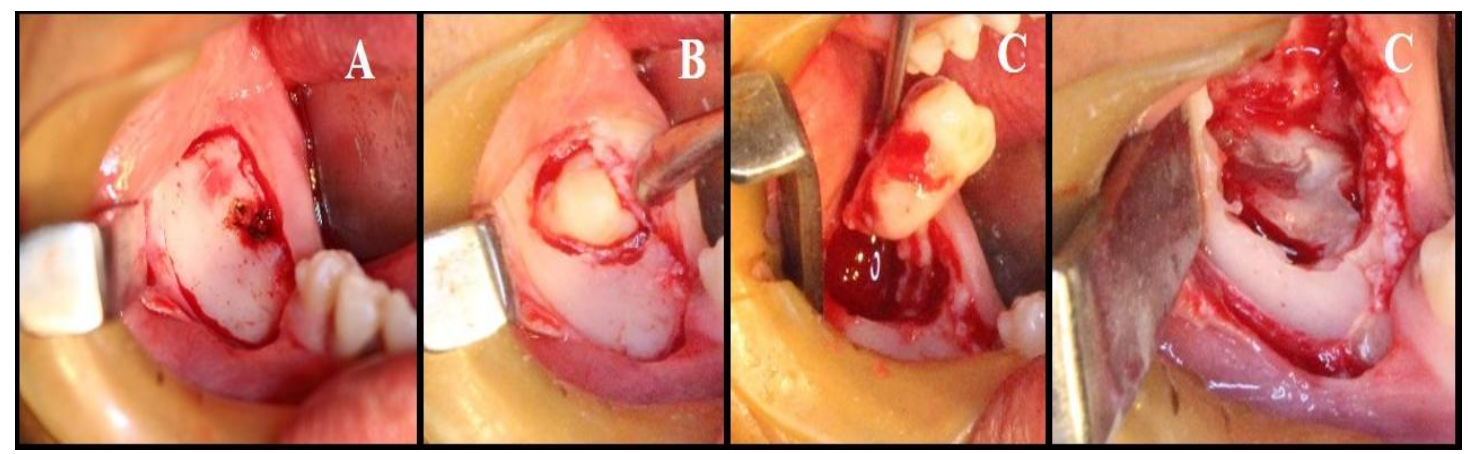

Fig-4: Exposure of right mandibular molar region showing bone covering the three molars; A-showing exposure of third molar; B- showing delivery of third molar from the socket; $\mathrm{C}$ - vertical sectioning of second molar

The first molar was left intact. Dental follicles with thick lining were removed along with all the surgically removed teeth and sent for biopsy (Fig-5). Platelet rich fibrin was placed in right and left mandibular extraction socket (Fig-6).

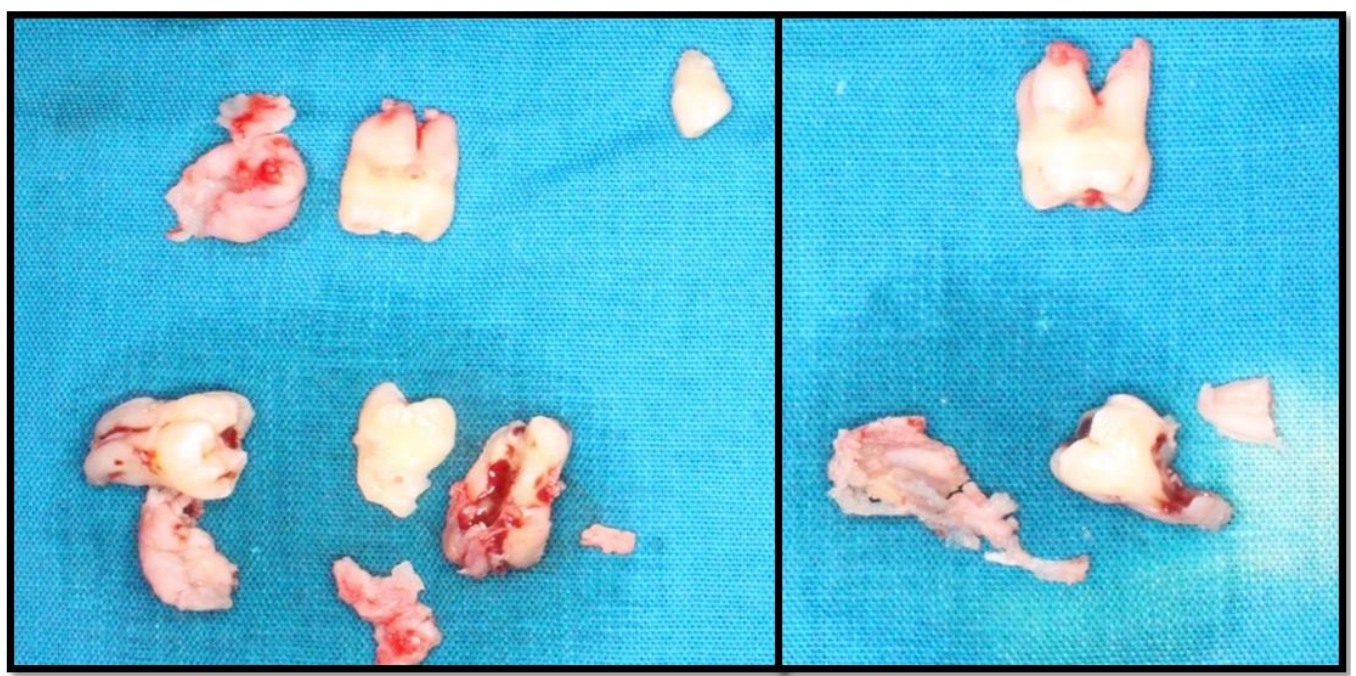

Fig-5: Showing surgically removed right mandibular second and third molars with the dental follicles, maxillary third molar and its dental follicle; left maxillary third molar and mandibular third molar with their follicles respectively

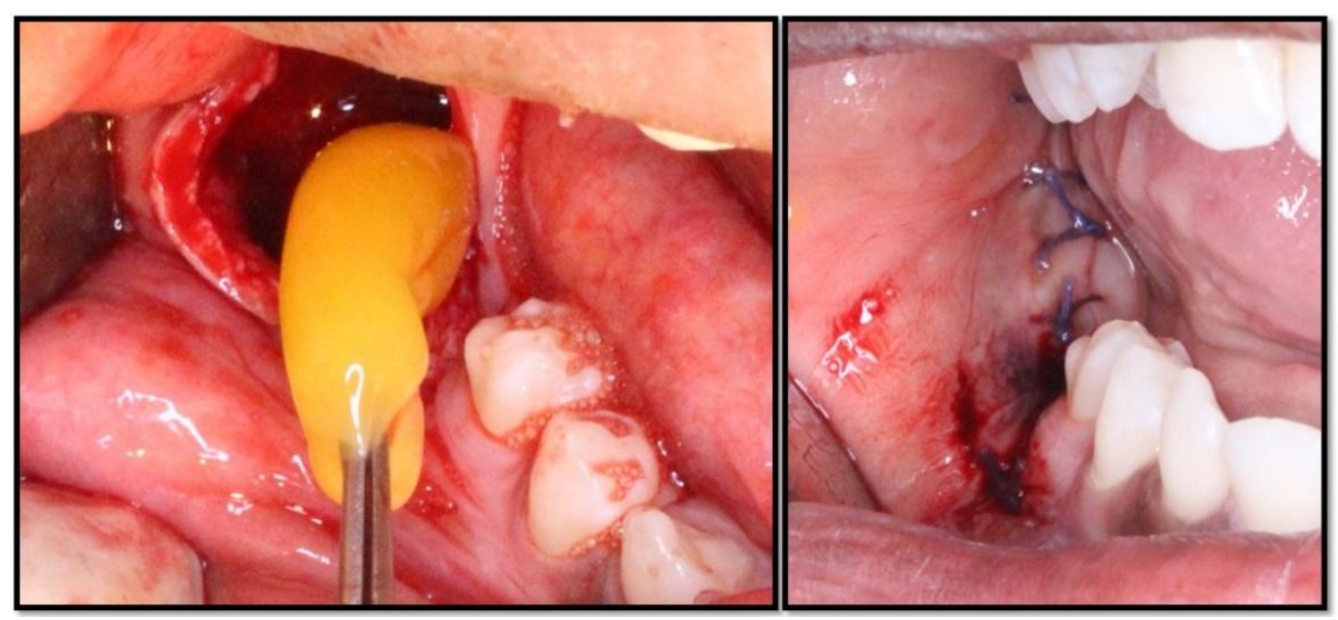

Fig-6: Placement of PRF in the right mandibular second and third molar socket; closure of the same 
Patient was kept on three days of parenteral antibiotics and discharged followed by two days of oral antibiotics, analgesics and anti-inflammatory drugs. Biopsy revealed multiple calcifying hyperplastic dental follicles. Healing was satisfactory with no complications at one month follow up (Fig-7). Patient has been advised for periodic regular follow up visit further.

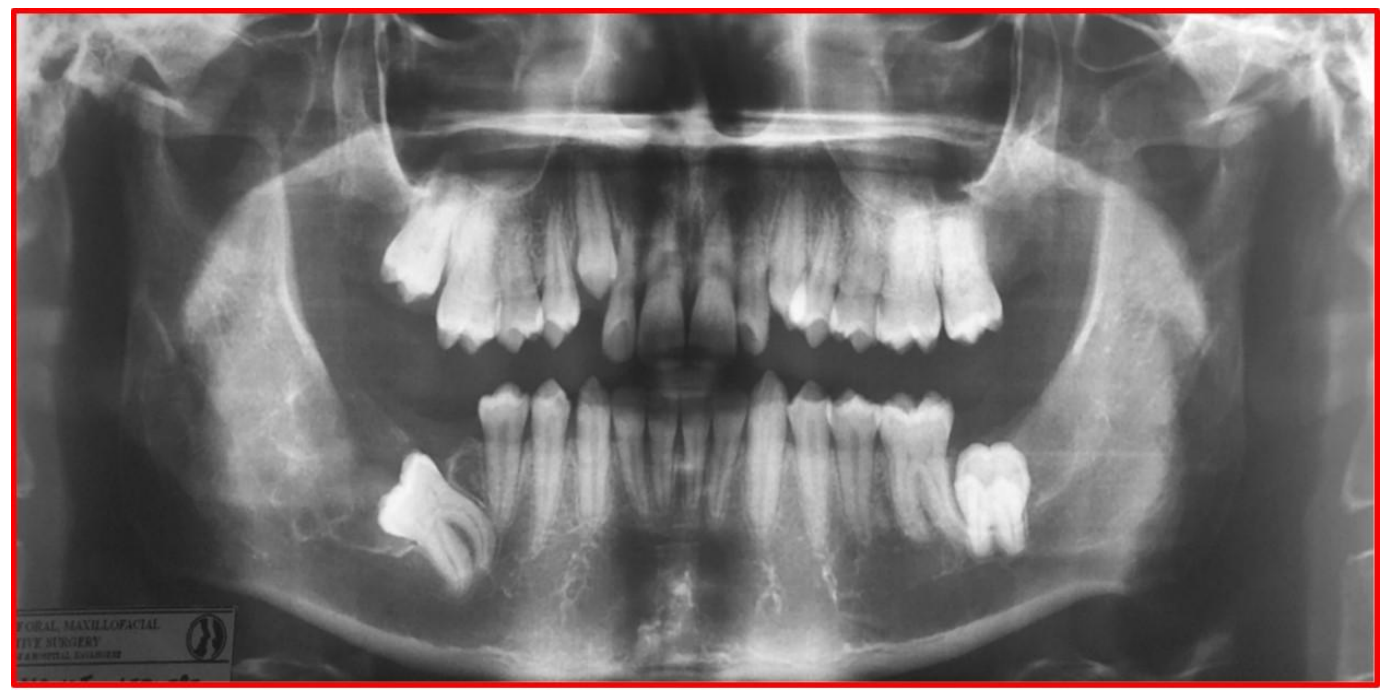

Fig-7: One month post operative radiograph

\section{DISCUSSION}

Van Hoof [2] in 1973 was the first to use the term "kissing molars" or 'rosette formation' and described the clinical case of bilateral inclusion in 31 year old mentally challenged patient. Robinson and colleagues [6] in 1991 reported the case of bilateral kissing molars involving the second and third mandibular molars in a 21 year old mentally and systemically healthy female patient. Nakamura and colleagues [5] reported a series of four cases of which three patients had multiple kissing molars and were also diagnosed with mucopolysaccharidosis. Nakamura concluded that this pathology could occur in patients who had mucopolysaccharidosis, although it can occur in isolation also. Mucopolysaccharidosis are a group of inherited disorders caused by the absence or accumulation of enzymes required to break down glycosaminoglycan which are required for maintenance and repair [7]. Impaction in inherited connective tissue diseases according to Menditti [8] is that they affect the connective tissue and then the bone surrounding the toothbud causing pathological changes that can lead to altered tooth eruption that lock, which leads to "rosetting". In the present case, because the patient presented clinical features such as hypertelorism, large nose, large lower lip and tongue, normal intellect for the specified age and had achieved appropriate growth milestones, the kissing molars could be associated with mucopolysaccharidosis or it could be an isolated case.

McIntyre [9] in 1997 described a symptomatic case of kissing molars in left side of mandible in a 19-year- old female. Since the patient developed trismus and dry socket after the surgery, the author emphasized on the importance of having an efficient surgical assessment and a treatment plan prior to the procedure and also the need to inform the patient about the possibilities of postsurgical complications that include paraesthesia. Koerner [10] in 2006 described a complicated case of symptomatic impacted molars in a 39-year-old man which required autogenous bone grafting and maxillomandibular fixation for four weeks. The author emphasized on the difficulties during surgical procedure of this type of impaction due to altered position of the teeth. He also stressed that one should consider close observation without surgery in asymptomatic patients. Krishnan ${ }^{11}$ in 2008 described a symptomatic case of kissing molars involving lower left mandibular second and third molars with an associated dentigerous cyst in a 36-year-old female patient. Rafael [12] in 2014 described a case of bilateral kissing molars in mandible with associated dentigerous cyst in a 33 year old male patient. Some authors have also explained the surgical technique used to remove the teeth and enucleation of the cyst. Impacted teeth may be associated with pathologies like infection, carious lesions, cysts and tumors [11].

Dentigerous cyst is the most common developmental odontogenic cyst in the jaw that presents as an asymptomatic unilocular radiolucency encircling the crown of an impacted tooth at the cementoenamel junction. It is difficult to differentiate between the small dentigerous cysts, healthy dental follicle or hyperplastic dental follicle with clinical observation. A clinical co-relation with histopathology findings and also macroscopic findings intra-operatively should be considered. In our case, histopathology revealed multiple calcifying hyperplastic dental follicles. 
Boffano and Gallesio [13] in 2009 described a case of unilateral impacted teeth with explanation of the surgical technique involving granular calcium sulphate filling of the socket in a 42-year-old male patient. Menditti [8] in 2015 reported three cases of kissing molars and explained theories of aetiopathogensis. Abnormal position of the tooth-bud from an early developmental stages of one or more normal lower permanent molars or fourth supernumerary tooth could lead to abnormal direction of development of their dental follicles which then could have abnormal eruption pathway causing two teeth to come together and form kissing molars. "Bone loss" theory [14] explained that a cystic formation resulted in bone loss on the mesial root of an impacted molar that may lead to the movement and rotation of teeth forming kissing molars. Similar theory can be applied when a fourth molar is present which acts as a pre- disposing factor for the bone loss adjacent to a third molar causing rotation of the molars involved. Recently Villalba [15] described in his study the association of kissing molars with hyperplastic dental follicle. Hyperplastic dental follicle (HDF) is a condition in which there is expansion of the follicular space of about 3-5 mm around the crown of an unerupted tooth in non-syndromic patients ${ }^{16}$. The histological analysis of the tissue of HDF shows great amounts of fibrous connective tissue and collagen fibres of the dental follicle, islands of odontogenic epithelium, mesenchymal multinucleated giant cells, and mineralized areas of calcification $[15,16]$.

Multiple calcifying hyperplastic dental follicles is another disorder in which enlarged dental follicles are present in association with multiple impacted teeth in a single patient histologically presenting fibrous tissue with numerous calcifications described by Sandler as Calcified-HDF for the first time and stated that this condition should be considered a distinct pathology. Its histological features are similar to HDF, but involves multiple teeth [17]. These findings are relevant with the findings in our case report; histopathology findings of the dental follicles associated with mandibular left second and third molar, mandibular right first, second and third molar, left maxillary third molar, right maxillary canine and third molar showed multiple calcifying hyperplastic dental follicles.

All the previously reported cases on kissing molars involve second and third molars or third and supernumerary fourth molars but literature has no reports involving first and second molars as seen in the our case report. Whenever a panoramic image assessment shows close relationship between impacted teeth and inferior alveolar nerve, CBCT is recommended to assess in all the three dimension, the exact position and distance of the impacted teeth from this important anatomical structure [18].

In the case of impacted two molars in a single follicle, great attention is needed in assessing the proximity of the teeth to the canal to avoid risk of morbidity. In our case the CBCT showed close proximity of the impacted right mandibular second molar to the canal that increased the difficulty of the procedure. There is also less literature explaining the management of the kissing molars. Since it is a rare phenomenon and not many reported cases, there is no established treatment approach or a specified surgical technique to deal with the impaction. However Van Hoof [2] in his article suggests that kissing molars can be evaluated according to its position. It should be considered as a type of impaction in a single follicular space that is associated with the crown of two molars with its roots in opposite directions [2].

Koerner [10] in his article states that it requires experience and great attention from the surgeon, demanding sometimes hospital settings with general anaesthesia and sometimes also need bone graft in the site of extraction. The treatment plan should be made depending on the age, position of the teeth, and relationship of the teeth with the inferior alveolar canal and careful evaluation of ensuing complications. Surgical removal should be considered when the teeth are in unfavourable position for eruption and orthodontic up righting. However when surgery is considered, the risk and morbidity of this procedure must be evaluated and informed to the patients. In the present case, the patient did not develop any intra-operative or post-operative complications. Some authors ${ }^{7}$ have reported complications like trismus and dry socket after the surgery, in our case however we have used Platelet Rich Fibrin in the sockets because of which the patient did not develop any complications. Socket grafting with PRF eliminates or reduces the incidence of dry socket [19], consistent with this study in our case also there was no incidence of dry socket.

The role of immunohistochemistry in mucopolysaccharidosis cannot be negated. However in our case we could not get the immunohistochemistry owing to patient consent \& follow up. Kissing molars carry precedence in that it is a rare entity, however occurrence of the same does not pose any significant problems. Though great attention is needed for evaluation of the case and the treatment in order to avoid damage to the nerve, the treatment per se in not difficult.

\section{CONCLUSION}

Rarity of occurrence of kissing molars has made it difficult to understand its pathology. There is no accepted explanation for its occurrence in syndromic patients diagnosed with mucopolysaccharidosis or why it occurs in nonsyndromic patients. Further studies and investigations are needed to understand the cause, develop treatment options and surgical techniques. 
Conflicts of Interest: None Declared

Source of Funding: None

Acknowledgements: None

\section{REFERENCES}

1. Fu, P. S., Wang, J. C., Chen, C. H., Huang, T. K., Tseng, C. H., \& Hung, C. C. (2012). Management of unilaterally deep impacted first, second, and third mandibular molars. The Angle Orthodontist, 82(3), 565-571.

2. Van Hoof, R. F. (1973). Four kissing molars. Oral Surgery, Oral Medicine, Oral Pathology, 35(2), 284.

3. Arjona-Amo, M., Torres-Carranza, E., Batista-Cruzado, A., Serrera-Figallo, M. A., Crespo-Torres, S., BelmonteCaro, R., ... \& Gutiérrez-Pérez, J. L. (2016). Kissing molars extraction: Case series and review of the literature. Journal of clinical and experimental dentistry, 8(1), e97-e101.

4. Yanik, S., Ayranci, F., İşman, Ö., Büyükçikrikci, Ş., \& Aras, M. H. (2017). Study of kissing molars in Turkish population sample. Nigerian journal of clinical practice, 20(6), 659-664.

5. Nakamura T., Miwa K., Kanda S., Nonaka K., Anan H., Higash S. et.al., Rosette formation of impacted molar teeth in mucopolysaccharidosis and related disorders. Dentomaxillofac Radiol 1992; 21(1): 45-9.

6. Robinson, J. A., Gaffney, W., \& Soni, N. N. (1991). Bilateral 'kissing'molars. Oral Surgery, Oral Medicine, Oral Pathology, 72(6), 760.

7. Melhern, R., Dorst, J. P., Scott Jr, C. I., \& McKusick, V. A. (1973). Roentgen findings in mucolipidosis III (pseudoHurler polydystrophy). Radiology, 106(1), 153-160.

8. Menditti, D., Laino, L., Cicciù, M., Mezzogiorno, A., Perillo, L., Menditti, M., ... \& Baldi, A. (2015). Kissing molars: report of three cases and new prospective on aetiopathogenetic theories. International journal of clinical and experimental pathology, 8(12), 15708.

9. McIntyre, G. (1997). Kissing molars: an unexpected finding. Dental update, 24(9), 373-374.

10. Koerner, K. R. (Ed.). (2006). Manual of minor oral surgery for the general dentist. Blackwell Publishing.

11. Krishnan, B. (2008). Kissing molars. British Dentel Journal, 204(6): 281-282.

12. Fortes, R. Z. S., Júnior, V. S., Modolo, F., \& Mackowiecky, E. (2014). Kissing molars: report of a case. Journal of Oral and Maxillofacial Surgery, Medicine, and Pathology, 26(1), 48-51.

13. Boffano, P., \& Gallesio, C. (2009). Kissing molars. The Journal of craniofacial surgery, 20(4), 1269-1270.

14. Gulses, A., Varol, A., Sencimen, M., \& Dumlu, A. (2012). A study of impacted love: kissing molars. Oral Health Dent Manag, 11(4), 185-188.

15. Villalba, L., Stolbizer, F., Blasco, F., Mauriño, N. R., Piloni, M. J., \& Keszler, A. (2012). Pericoronal follicles of asymptomatic impacted teeth: a radiographic, histomorphologic, and immunohistochemical study. International journal of dentistry, 2012, 1-6.

16. Jamshidi, S., Zargaran, M., \& Mohtasham, N. (2013). Multiple calcifying hyperplastic dental follicle (MCHDF): a case report. Journal of dental research, dental clinics, dental prospects, 7(3), 174-176.

17. Schmitd, L. B., Bravo-Calderón, D. M., Soares, C. T., \& Oliveira, D. T. (2014). Hyperplastic dental follicle: a case report and literature review. Case reports in dentistry, 2014, 1-7.

18. Maegawa, H., Sano, K., Kitagawa, Y., Ogasawara, T., Miyauchi, K., Sekine, J., \& Inokuchi, T. (2003). Preoperative assessment of the relationship between the mandibular third molar and the mandibular canal by axial computed tomography with coronal and sagittal reconstruction. Oral Surgery, Oral Medicine, Oral Pathology, Oral Radiology, and Endodontology, 96(5), 639-646.

19. Kattimani, V. S., Lingamaneni, K. P., Kreedapathi, G. E., \& Kattappagari, K. K. (2019). Socket preservation using eggshell-derived nanohydroxyapatite with platelet-rich fibrin as a barrier membrane: a new technique. Journal of the Korean Association of Oral and Maxillofacial Surgeons, 45(6), 332-342. 